\title{
Young children with fetal alcohol spectrum disorder - communication profiles
}

\author{
Mari de Beer \\ Alta Kritzinger \\ Ursula Zsilavecz \\ Department of Communication Pathology, University of Pretoria
}

Correspondence to: $M$ de Beer (mari.debeer@up.ac.za)

\begin{abstract}
The aim of the article is to describe the communication profiles of five young children with fetal alcohol spectrum disorder (FASD) from 4 to 58 months of age. A collective case-study design following a quantitative and descriptive approach was used to describe the communication profiles of the participants. The results are described according to the participants' case histories and a four-level early communication assessment framework. The significant findings were that all participants were in foster care, and presented with incomplete case histories, general developmental delays and delays regarding all aspects of their communication abilities. An increase in the severity of the spectrum disorder across the participants' combined communication profiles was also identified. Participants presented with complex multiple neurodevelopmental needs that should be viewed within a developmental systems and ecological framework. The importance of early identification, diagnosis and assessment of infants and young children prenatally exposed to alcohol, the identification of precursors to communication impairment at a very early age, and the need for individualised early communication intervention to improve developmental outcomes within a family-centred approach are discussed. Suggestions for future research to accumulate knowledge about FASD in the field of early communication intervention are made.
\end{abstract}

Keywords: fetal alcohol spectrum disorder, communication profile, early communication intervention

According to research of the past 10 years, South Africa has the highest prevalence of fetal alcohol spectrum disorder (FASD) in the world (Viljoen, Craig, Hymbaugh, Boyle \& Blount, 2001a). FASD has long been identified as the leading cause of preventable mental disability in the USA (Antoniadis \& Daulton, 1992; Sparks, 2000). Despite the high prevalence of FASD in children and the resulting need for services, appropriate identification and management of genetic and congenital disorders are critically lacking in South Africa (Ntsabula, 2001). FASD is a lifelong congenital disorder characterised by multiple neurodevelopmental disabilities, including enduring learning, communication and behaviour deficits (Carmichael Olson, Jirikowic, Kartin \& Astley, 2007). The term 'spectrum' is currently preferred to 'syndrome', since it is more realistic to describe the condition as a range of disorders that vary from mild to severe, than 'syndrome' which only refers to a collection of co-occurring symptoms; 'spectrum' is therefore more descriptive than 'syndrome'.

Services to families and communities with children with FASD are still insufficient. Consequently, the high prevalence of children with FASD in South Africa provides impetus to health service providers to be competent in the areas of prevention of FASD, early identification, diagnosis, assessment and subsequent intervention.

Since speech-language therapy forms part of the services that should be provided to families of children with genetic and congenital disorders, and considering the high prevalence of FASD in South Africa, it can be expected that the number of children with FASD in the caseload of speech-language therapists (SLTs) will increase. It appears, however, that only a limited description of the communication development of young children with FASD is available in the relevant literature (Sparks, 2000).

It is therefore useful to document cases of FASD in order to contribute to the limited knowledge base of the spectrum disorder, to use the information for prevention, early identification and individualised early intervention services, and to facilitate family-to-family contact for support networks. Knowledge about FASD is particularly important for SLTs and audiologists, since limited data exist on the early communication development, feeding and hearing concerns of infants and young children with FASD.
Studies conducted in the Western Cape region of South Africa indicate that the identification and diagnosis of children with FASD or fetal alcohol effects (FAE) (children not complying with the full diagnostic criteria of FASD) occur late, mainly at school entry (Viljoen, Croxford, Gossage, Kodituwakku \& May, 2001b). An investigation into the early communication development of children diagnosed with FASD may provide additional guidelines for the early detection and appropriate early management of communication disorders in this vulnerable population of young children. Early detection may ensure that infants and young children with FASD and their families enjoy the full benefits of early communication intervention (ECI) in the form of caregiver training and support. However, for ECI to be successful, a comprehensive understanding of FASD and the diagnostic criteria of this condition is essential.

The diagnostic criteria as formulated by Carmichael Olson et al. (2007) for identifying FASD in a child are supplied in Table I.

The process of diagnosing FASD in a child requires a visual examination of the physical features of the child, obtaining a case history, and conducting a behavioural assessment of the child. The visual examination is conducted by a medical specialist (i.e. dysmorphologist, geneticist or paediatrician), who usually also obtains the case history in order to make the diagnosis. Various multidisciplinary team members, including a clinical psychologist, social worker, SLT, occupational therapist or physiotherapist, can conduct assessments to arrive at a team diagnosis; however, the tendency is for medical specialists to make the diagnosis (Clarren \& Astley, 1997).

Once the assessments have been conducted, all four main diagnostic criteria (see Table I) must be present in order to diagnose a child with FASD. However, children with FASD may also display more symptoms than those listed in the four main diagnostic criteria. When fewer than four diagnostic criteria are present, children may be diagnosed with FAE. FAE is found in children who were prenatally exposed to alcohol and 'are at increased risk of attention impairment and other cognitive difficulties, growth impairment, difficulties in fine and gross motor skills and speech-language problems' (Carmichael Olson \& Burgess, 1997: 114). Since children with FAE display a subset of the symptoms that are associated with the diagnostic criteria of FASD, the entire 
Table I. Diagnostic criteria

Diagnostic terminology
1. Fetal alcohol syndrome (FAS)

(all 4 diagnostic characteristics required for diagnosis)
Diagnostic characteristics

- Prenatal and/or postnatal growth deficiency

- Central nervous system (CNS) impairment

- Cluster of craniofacial dysmorphology

- Confirmed prenatal alcohol exposure
Description

- Height or weight less than the 10th percentile for gestational age at birth or later in life

- Evidence of structural, functional or neurological brain impairment

- Small palpebral fissures (eye slits), thin upper lip, smooth philtrum

- Reliable evidence of maternal alcohol consumption. If evidence not obtained, it is not deemed necessary if the cluster of facial features is fully present
2. Partial fetal alcohol syndrome (PFAS) (only 3 diagnostic characteristics required for diagnosis)

3. Alcohol-related neurodevelopmental disorder (ARND) (only 2 diagnostic characteristics required for diagnosis)
- Growth deficiency

- Cluster of craniofacial dysmorphology

- CNS impairment

- CNS impairment

- Confirmed prenatal alcohol exposure

Source: Carmichael Olson, Jirikowic, Kartin \& Astley, 2007.

population of children who have FASD and FAE may therefore display great variability in symptoms on a continuum ranging from mild to severe impairment (Carmichael Olson \& Burgess, 1997).

According to Dittmer and Lentz (2004), the fetal pathophysiology brought about by the consumption of alcohol during pregnancy is expressed as follows: alcohol crosses the placenta and rapidly reaches the fetus. Unimpeded bidirectional movement of the alcohol between the mother and the fetus takes place. The fetus is dependent on maternal hepatic detoxification, since the activity of alcohol dehydrogenasis in the fetal liver is less than $10 \%$ of that in the adult liver. The amniotic fluid acts as a reservoir for alcohol, resulting in prolonged fetal exposure and alteration of fetal development in the following ways:

- disruption of cellular differentiation and growth

- disruption of DNA and protein synthesis

- inhibition of neuronal and glial cell migration

- deprivation of intra-uterine nutrients, which has a direct effect on fetal growth

- causation of a chronic state of fetal hypoxia (hypoxia refers to an oxygen shortage in the tissue).

Prenatal exposure to alcohol may therefore have pervasive effects on the fetus, and the visible features of these effects can be diagnosed at birth. According to Abkarian (1992) the recognisable physical appearance of children with FASD is more pronounced during infancy and may aid in early diagnosis.

The process of arriving at a positive diagnosis of FASD, however, does not provide an adequate basis for a prognosis regarding the level of cognitive impairment and the degree of developmental delay in a specific child. A comprehensive individual communication assessment may assist in determining each child's profile of strengths and weaknesses, as well as protective and risk factors in the family environment, before prognostic predictions can be ventured in the diagnostic process. However, a conclusive diagnosis of FASD cannot always be established in young children (Clarren, Carmichael Olson, Clarren \& Astley, 2000), since manifestation of deficits or impairments in the cognitive and other developmental domains are not as apparent early in life as when the child is older. The accurate prognosis of FASD is further complicated by the variable effects of alcohol in a pregnant woman, the degree of FASD and the effect of intervention on the individual (Clarren et al., 2000).

Appendix A provides additional characteristics of children with FASD and FAE to those described in the diagnostic criteria. The summarised information in Appendix A emphasises the extensive physical, medical, developmental and behavioural characteristics of children with FASD. The review of the literature, together with Appendix A, provides a comprehensive description of known symptoms that may assist SLTs and other health professionals to identify and assess young children with FASD.

The broad spectrum of symptom combinations demonstrates the potential far-reaching effects of prenatal alcohol exposure on fetal development (Clarren et al., 2000). Different variables may also interact with one another. Variables such as dosage of exposure, duration of exposure, gestational timing, genetic characteristics, drinking pattern, type of beverage, individual variation in alcohol metabolism, nutritional status of the mother and the varied susceptibility of the fetus contribute to the severity of symptoms that a child prenatally exposed to alcohol may display (Colangelo \& Jones, 1982). The symptoms are present in a continuum and may range from clearly within the normal range to distinct features of FASD (Clarren et al., 2000). As displayed in Appendix A and according to Steinhaussen, Willms, Winkler Metzke and Spohr (2003), children with FASD present with a behavioural phenotype. A behavioural phenotype refers to a biological disorder causing a characteristic pattern of motor, cognitive, linguistic and social deficits that can be consistently associated with FASD.

Research has shown that many children with FASD display unusual and varied language impairments, but this area has received limited attention among researchers (Carmichael Olson \& Burgess, 1997). To facilitate early identification of children with FASD, a comprehensive communication profile outlining the peculiar speech and language impairments of these children would be helpful to clinicians to guide disorder-specific assessment and intervention. Since the present study focused on the communication abilities of young children with FASD, an overview of the known communication impairments as described in the literature is provided in Appendix B. The literature study of communication impairments of children with FASD served as a basis to compile a comprehensive early communication assessment protocol to be used as data collection material in the empirical study. The literature review covers an asset-based assessment approach, the impact of substance abuse on family interaction patterns, and specifically parent-child interaction, the application of an ecological approach to assessment, and ECI for young children with FASD.

The current perspective in ECI service delivery is to emphasise an asset-based approach. Every individual has a profile of strengths and weaknesses, and appropriate ECI services can capitalise on the strengths and may ameliorate some of the weaknesses in children with FASD. Young children affected by FASD do not display the negative sequelae of prenatal exposure to alcohol only, but may also demonstrate certain positive characteristics, such as being socially engaging, interested in others, talkative - despite numerous expressive language problems - 
and affectionate (Carmichael Olson \& Burgess, 1997). Protective factors such as child resilience and foster care placement may be present, but the risks in the social environment, family circumstances and poor attachment with the primary caregiver may be of great concern in children with FASD. Often underscored in the literature, poor mother-child attachment adds to communication impairment, since the required developmental stimulation is not provided. Appropriate attachment with the primary caregiver is viewed as a foundation for optimal language development, which also forms the basis for cognitive development (Sparks \& Gushurst, 1997).

In the case of children with FASD/FAE, the personal characteristics of a substance-abusing parent, as well as those of another present or absent parent/caregiver, will influence the family patterns. According to Guralnick (1997), family patterns will be influenced by the quality of parent-child transactions, family-orchestrated child experiences and the health care and safety provided by the family to the child. An additional risk factor is the unstable home environments of most children with FASD (Vig, Chinitz \& Shulman, 2005). Children with FASD are often placed in foster care because of poor social circumstances. Vig et al. (2005) report that young children, even those without FASD, who have been removed from their biological families and placed in foster care, are at a significant risk for poor developmental outcomes.

The diagnosis of FASD in a child creates additional stressors regarding information needs (regarding what FASD entails and prognostic information), interpersonal and family distress (poor coping skills and maladaptive parenting), resource needs (limited access to appropriate EI services) and confidence threats (feeling inadequate regarding childrearing practices for a child with special needs) (Guralnick, 1997). The conceptual model of Guralnick (1997), as well as an ecological approach (Gabarino \& Ganzel, 2000), may be used to understand the multitude of factors likely to impinge on the development of the child with FASD/ FAE. These theoretical approaches are founded on the understanding that social units such as a family do not act in isolation, but interact both between and within systems. A strong interplay is present between aspects relating to nature and nurture, where interaction takes place between the characteristics of the child with FASD and the environment in which the child is raised (Garbarino \& Ganzel, 2000). Therefore, reverberations occur across all the areas of the child's development. For this reason, ECI for children with FASD should focus on caregiver training as well as information giving and support. Furthermore the focus should also be on the highest possible degree of development of the children's stronger abilities in order to compensate for the impairments that are present. Empowering affected children and their families with coping strategies which may facilitate improved caregiverchild interaction and child development should also form an integral part of ECI focus in this population. Viljoen and colleagues (2001) identified specific South African communities with a high incidence of children with FASD (Department of Health, 2001). This highlights that health professionals should be aware that some communities are more at risk for the prevalence of FASD than others, to be able to provide more appropriate services in those communities. Although no national FASD survey has been conducted yet, it appears that specific communities will play an integral role regarding the prevention and identification of FASD, and the facilitation of access to ECI services for affected children and their caregivers.

The rationale for this study was outlined in the existing need for local data and a communication profile of infants and young children with FASD. The research question that emerged from the rationale was: What are the nature of communication abilities and impairments, as well as the environmental circumstances of a group of infants and young children with FASD in the South African context?

\section{Method \\ Main aim}

The main aim of the study was to present distinctive communication profiles of a small number of infants and young children diagnosed with FASD.

\section{Objectives}

- To describe the developmental histories of the infants and young children with FASD.

- To provide a description of their family/caregiving contexts.

- To describe the communication functioning of each participant with FASD in order to present individual communication profiles as well as a combined developmental trend of the children.

\section{Research design}

A collective case-study design following a quantitative approach was selected (Leedy \& Ormrod, 2005). Descriptive statistics were used to describe the communication profiles of the participants who were selected by means of a purposive sampling procedure. Owing to the limited number of participants that could be recruited for the study, the participants were too heterogeneous in age to describe as a group. The research design allowed the researcher to study two sets of participants, namely the parents/caregivers, as well as the infants and young children with FASD, as case studies.

A cross-sectional observational method was used, as the two groups of participants were only studied once. Child participants from different age groups were sampled and compared (Leedy \& Ormrod, 2005) in order to indicate trends in their development.

\section{Ethical considerations}

Permission to conduct the study was obtained from the Research Ethics Committee of the Faculty of Humanities, University of Pretoria, and the Gauteng Department of Health. Confidentiality applies to all studies, but because of the sensitive nature of parental abuse of alcohol and its effect on children, special care was taken to keep participants' identities confidential. All caregiver participants gave informed consent to participate in the study. Full disclosure of assessment results was provided to the caregivers and appropriate early intervention referrals were made. The assessment results were discussed within an asset-based approach with the caregivers, linking the findings to FASD and indicating future implications of the young child's strengths and weaknesses and how best to address areas of need.

\section{Research participants}

The main inclusion selection criteria for the child research participants in the study were as follows:

- The child participants had to have a diagnosis of FASD that included a confirmed history of prenatal exposure to alcohol or other drugs, growth retardation, central nervous system dysfunction and FASDspecific dysmorphic features (Carmichael Olson et al., 2007).

- The ages of the participants had to be between 0 and 5 years to comply with the aims of the study, i.e. to investigate a group of infants and young children eligible for ECI.

- The participants had to be in the greater Pretoria area in Gauteng. This would make access to participants easier and therefore more time- and cost-effective for the researcher.

The selection criteria for the parents/caregivers were:

- Caregivers/parents of the participants had to provide informed consent (verbal or written) to participate in the research study themselves and for the child participant to participate.

- The caregivers/parents' and children's first or second language had to be Afrikaans or English, since the researcher is proficient in these two languages.

Despite the high prevalence of FASD in South Africa, participant recruitment difficulties were experienced in some of the at-risk communities identified in Gauteng, with resistance to research involvement with no perceived benefit for the community. However, 
free ECI services were offered at an ECI clinic if the child participants required the services. This free service offer was explained to the community sites before commencement of the research. Furthermore it came as a surprise that no biological parents took part in the study, possibly because of the stigmatisation attached to FASD in their communities.

\section{Description of the foster and adoptive parents, and child participants}

The seven adult participants in the study were foster parents of the participants (5 mothers and 2 fathers). Two fathers participated with their wives. Knowledge and information regarding the biological parents were scant or unknown in all the cases and will be presented as part of the results of the study. The participants reported that the social workers did not provide detailed background information on the biological parents to the foster parents. Five of the seven foster parents had tertiary education and all the fathers were employed. The composition of the foster families revealed a relatively small number of children ( 1 - 3 children). Werner (2000) reported that small families appeared more resilient as a result of better stimulation and more attention being provided to the children by the parents. It could therefore be possible that the foster and adoptive families provided the participants with stimulating environments, which can be considered an asset. The caregivers were a data source providing the case history and developmental information regarding each participant.

\section{The characteristics of the child participants are summarised in Table II.}

All the participants had spent various periods in the foster care system. Additional characteristics were that gender was not equally divided between participants, but could be regarded as incidental distribution. It is further evident from Table II that the child participants had a wide age range. Four of the five participants had only been diagnosed with FASD after being placed in foster care. According to Vig et al. (2005), children placed in the foster care system in the USA are usually thoroughly examined by medical specialists and referred to related team members such as therapists, when necessary. This could be a possible explanation of diagnosis only being made after placement in the foster care system in South Africa. A lack of attendance to medical or developmental needs in the child's biological family could have been present because of poverty, lack of accessible services and alcohol abuse in the family (Vig et al., 2005). The age of diagnosis in participants 1, 2 and 5 indicated that very early diagnosis of FASD is possible, although not prevalent according to Viljoen et al. (2001a).

\section{Materials}

The materials used during data collection for the study were:

- Interview schedule with five categories: general information, prenatal and birth history, medical history, developmental history and parental concerns and needs.

- A communication assessment protocol, adapted from Kritzinger and Louw (2002) to evaluate specific characteristics of FASD.

The comprehensive four-level early communication assessment framework (Kritzinger \& Steenkamp, 2006), as used by the Clinic for High-Risk Babies (CHRIB) assessment team at the University of Pretoria, provided a theoretical basis for assessing a child and family affected by FASD. The first level includes screening checklists and procedures to describe the physical appearance of the child that may relate to genetic anomalies, the prenatal and perinatal history, and to ascertain the child's sensory abilities. The second level focuses on the in-depth assessment of communication functioning and feeding skills of the child. Level three involves the screening of general developmental domains, such as cognitive, gross and fine motor development. The function of screening general developmental areas is also to determine the need for referral to specialised assessment by other disciplines such as occupational therapy. The fourth level of the four-level early communication assessment framework of Kritzinger and Steenkamp (2006) renders a description of all the risk and resilience identified in the child and family (Werner, 2000). The research methods utilised were a structured interview with the caregiver, assessment of the child and observation of caregiver-child interaction during the assessment section.

\section{Procedures \\ Validity and reliability}

The reliability and validity of the study procedures were enhanced by conducting a pilot study on one child with FASD and the family (Leedy \& Ormrod, 2005). The pilot study was deemed successful, because sufficient data were collected on one participant and would therefore be adequate for an in-depth description of the participants of the main study. No changes were required for the structured interview or the assessment protocol. Within the assessment protocol the assessment instruments were deemed applicable and the sequential order of assessment procedures was confirmed as appropriate. The different assessment instruments and procedures comprising the early communication assessment protocol have already been used in previous ECI research studies and proven to be valid (Kritzinger \& Louw, 2002; Kritzinger \& Steenkamp, 2006). To increase the reliability of data collection and analysis, a second rater was used for the analysis of mother-child interaction. The researcher and second rater analysed the videos independently and discussed points of difference until agreement could be found.

The internal validity was addressed through triangulation (De Vos, 2002). The researcher's observations were enhanced by the parental report, video and audio recordings and a second rater for the analysis of mother-child interaction. The content validity was addressed by ensuring that the research materials corresponded with interview schedules, assessment protocols and theoretical frameworks in the field of ECI research and in agreement with the aims of the study. Face validity was addressed by using a patient/caregiver information leaflet, structuring the interview schedule and assessment protocol in a logical way and using appropriate language and terminology - all of which was tested in a pilot study (Leedy \& Ormrod, 2005). Construct validity required that the interview schedule and assessment protocol contain only pertinent questions and assessment tasks that relate to the study's objectives (Leedy \& Ormrod, 2005). The findings of the study cannot be generalised or transferred to the entire FASD population because of the small, unrepresentative sample utilised in this study (De Vos, 2002).

\section{Data collection procedures and analysis}

The data were collected during separate interview and assessment sessions for the five participants. A structured interview with the foster parent/s was conducted to obtain a comprehensive case history and developmental overview of the child participant. The assessment session consisted of a play-based assessment and a section for parent-child interaction. The rooms of a private speech-language therapy practice

Table II. Description of child participants $(N=5)$

\begin{tabular}{lllllll}
\hline Characteristic & Participant 1 & Participant 2 & Participant 3 & Participant 4 & Participant 5 \\
Chronological age & 4 months & 18 months & 35 months & 51 months & 58 months \\
Age when diagnosed with FASD & At birth & 15 months & 28 months & 48 months & At birth \\
$\begin{array}{l}\text { Age when placed in foster care system } \\
\text { Age when adopted }\end{array}$ & 5 weeks & 12 months & 26 months & 24 months & At birth \\
N/A & N/A & N/A & During first \\
Gender & Male & Male & Female & Female & Male of \\
\hline
\end{tabular}


and the Department of Communication Pathology at the University were used for the sessions. Data were recorded on the assessment forms, developmental levels were determined according to the scoring procedures of the different assessment instruments, and the results were summarised and represented in tables and graphs.

\section{Results and discussion}

Objectives 1 and 2: To describe the developmental histories of the five participants with FASD and their family/ caregiving contexts

According to Table III certain trends emerged from the case histories of the five participants. All participants had a confirmed diagnosis of FASD, indicating that early diagnoses had occurred despite reports by Viljoen et al. (2001b) that in South Africa children were mostly identified at school entry level. However, the results cannot be generalised as it was a selection criterion to include participants already diagnosed with FASD.

Four of the five participants were living in foster care, one had already been adopted, and one adoption was in progress. Children with FASD in the foster care system in South Africa may be more accessible for research than children living with their biological families. Placement in foster care resulted in improved circumstances, such as stability, safer environments, and improved access to health care services for the child participants compared with their biological family settings, which were characterised by chaotic lifestyles, posing health and safety risks and exposing the child participants to maltreatment. The foster and adoptive parents displayed a lack of information regarding early intervention services, specifically ECI services. The unknown histories of perinatal and postnatal development were characteristic of the participants. The incomplete or lost histories of the period before placement in foster care may be a concern for the participants' sense of identity as they grow older. Furthermore, reliable description of risks such as low birth weight and preterm birth may not be possible.

The participants were diagnosed by a single medical professional in a public hospital, without comprehensive team involvement, except for the social worker who was involved in follow-up and foster care placement.

The participants displayed problems regarding early self-regulatory behaviour and feeding. Recurrent otitis media and tonsillitis suggest that they may have compromised immune systems. The foster and adoptive parents reported that the participants displayed delayed achievement of developmental milestones in different developmental domains, but mostly in listening, speech, language and motor development. The participants were described as talkative and active children by their caregivers. The

Table III. Summary of the participants' case histories as reported by the foster and adoptive parents $(N=5)$

\begin{tabular}{|c|c|c|c|c|c|}
\hline $\begin{array}{l}\text { Categories } \\
\text { 1. Biological families }\end{array}$ & $\begin{array}{l}\text { Participant } 1 \\
\text { Age: } 4 \text { mo., male }\end{array}$ & $\begin{array}{l}\text { Participant } 2 \\
\text { Age: } 18 \text { mo., male }\end{array}$ & $\begin{array}{l}\text { Participant } 3 \\
\text { Age: } 35 \text { mo., female }\end{array}$ & $\begin{array}{l}\text { Participant } 4 \\
\text { Age: } 53 \text { mo., female }\end{array}$ & $\begin{array}{l}\text { Participant } 5 \\
\text { Age: } 58 \text { mo., male }\end{array}$ \\
\hline Parental ages & $\begin{array}{l}\text { Mother: } 25 \\
\text { Father: Not available }\end{array}$ & Not available & $\begin{array}{l}\text { Mother: } 27 \\
\text { Father: } 28\end{array}$ & Not available & $\begin{array}{l}\text { Mother: } 35 \\
\text { Father: Not available }\end{array}$ \\
\hline Parental education & Not available & Not available & $\begin{array}{l}\text { Both in special } \\
\text { needs school }\end{array}$ & Not available & Not available \\
\hline Parental occupations & $\begin{array}{l}\text { Mother: Housewife } \\
\text { Father: Gardener }\end{array}$ & $\begin{array}{l}\text { Mother: Veterinary } \\
\text { assistant }\end{array}$ & $\begin{array}{l}\text { Mother: Shop assistant } \\
\text { Father: Gardener }\end{array}$ & Not available & Not available \\
\hline $\begin{array}{l}\text { Socio-economic status } \\
\text { (SES) } \\
\text { Siblings with FASD } \\
\text { Home language } \\
\text { Substance abuse }\end{array}$ & $\begin{array}{l}\text { Low SES } \\
\text { No } \\
\text { Sotho } \\
\text { Alcohol abuse } \\
\text { Poly-drug use }\end{array}$ & $\begin{array}{l}\text { Middle class } \\
\text { No } \\
\text { English } \\
\text { Alcohol abuse }\end{array}$ & $\begin{array}{l}\text { Middle class } \\
\text { Yes } \\
\text { Afrikaans } \\
\text { Alcohol abuse }\end{array}$ & $\begin{array}{l}\text { Low SES } \\
\text { Yes } \\
\text { Afrikaans } \\
\text { Alcohol abuse } \\
\text { Cigarette use }\end{array}$ & $\begin{array}{l}\text { Middle class } \\
\text { Not available } \\
\text { Afrikaans } \\
\text { Alcohol abuse } \\
\text { Cigarette use }\end{array}$ \\
\hline $\begin{array}{l}\text { 2. Foster family } \\
\text { Home language } \\
\text { Nursery school } \\
\text { attendance }\end{array}$ & $\begin{array}{l}\text { Afrikaans } \\
\text { Double-medium } \\
\text { crèche }\end{array}$ & $\begin{array}{l}\text { Afrikaans \& English } \\
\text { At home }\end{array}$ & $\begin{array}{l}\text { Afrikaans } \\
\text { Afrikaans nursery } \\
\text { school }\end{array}$ & $\begin{array}{l}\text { Afrikaans \& English } \\
\text { Double-medium } \\
\text { nursery school }\end{array}$ & $\begin{array}{l}\text { Afrikaans } \\
\text { Afrikaans nursery } \\
\text { school }\end{array}$ \\
\hline 3. Perinatal history & $\begin{array}{l}\text { Full term, NICU } \\
\text { Natural birth } \\
\text { Breastfed by biological } \\
\text { mother }\end{array}$ & Not available & Not available & $\begin{array}{l}\text { Not available } \\
\text { Bottle fed }\end{array}$ & $\begin{array}{l}\text { Full term } \\
\text { Natural birth } \\
\text { Bottle fed }\end{array}$ \\
\hline Birth weight & $2400 \mathrm{~g}$ & Not available & Not available & Not available & $2805 \mathrm{~g}$ \\
\hline $\begin{array}{l}\text { 4. Early behavioural } \\
\text { characteristics }\end{array}$ & $\begin{array}{l}\text { Irritable } \\
\text { Inconsolable } \\
\text { Very active }\end{array}$ & Very active & Not available & $\begin{array}{l}\text { Very active } \\
\text { Friendly }\end{array}$ & $\begin{array}{l}\text { Irritable } \\
\text { Inconsolable } \\
\text { Very quiet }\end{array}$ \\
\hline 5. Medical history & $\begin{array}{l}\text { High temperature after } \\
\text { inoculation } \\
\text { Bronchiolitis } \\
\text { Eczema } \\
\text { Possible lactose } \\
\text { intolerance } \\
\text { GORD }\end{array}$ & $\begin{array}{l}\text { Chronic middle ear } \\
\text { infections } \\
\text { Grommets } \\
\text { Chronic tonsillitis } \\
\text { Gastro-enteritis } \\
\text { Strabismus }\end{array}$ & $\begin{array}{l}\text { Tonsillitis } \\
\text { Regular colds \& flu }\end{array}$ & $\begin{array}{l}\text { Chronic middle-ear } \\
\text { infections } \\
\text { Grommets } \\
\text { Chronic tonsillitis } \\
\text { Tonsillectomy } \\
\text { Regular colds \& flu, sinusitis } \\
\text { Colic } \\
\text { Reconstruction of cleft } \\
\text { palate } 27 \text { m } \\
\text { ADHD medication }\end{array}$ & $\begin{array}{l}\text { One episode of high } \\
\text { temperature } \\
\text { Tonsillitis } \\
\text { Regular colds \& flu }\end{array}$ \\
\hline 6. History of & Emesis after birth & & & & \\
\hline feeding problems & $\begin{array}{l}\text { Breast-bottle } \\
\text { transition problems }\end{array}$ & No & No & $\begin{array}{l}\text { Feeding difficulties associated } \\
\text { with cleft palate }\end{array}$ & No \\
\hline 7. Current feeding status & $\begin{array}{l}\text { Emesis, excessive } \\
\text { drooling }\end{array}$ & Age appropriate & Age appropriate & Age appropriate & Age appropriate \\
\hline 8. Hearing difficulties & No & Yes & No & No & No \\
\hline 9. Behavioural & Sleep difficulties & No & Limited attention & Limited attention & Yes \\
\hline
\end{tabular}

difficulties

$\mathrm{NICU}=$ neonatal intensive care unit $; \mathrm{ADHD}=$ attention deficit hyperactivity disorder; $\mathrm{GORD}=$ gastro-oesophageal reflux disease 
characteristics in Table III are also described in the literature as typical of children with FASD (Carmichael Olson et al., 2007).

The trends observed in the case histories of the five participants may serve as guidelines for SLTs in clinical practice to obtain as comprehensive a case history as possible if a child is suspected of FASD. These observed trends may also be of value in the promotion of effective interdisciplinary team management of young children with FASD.

\section{Objective 3: Communication profiles of participants}

A classification of severity of delay was implemented to summarise each participant's results in table format. The severity classification was derived from Rossetti (2006) and the Arizona Revised Statutes 15-761 (2010). A mild developmental delay is defined as the child performing 6 months below chronological age expectations in developmental skill mastery, measured against the norms of typically developing children.

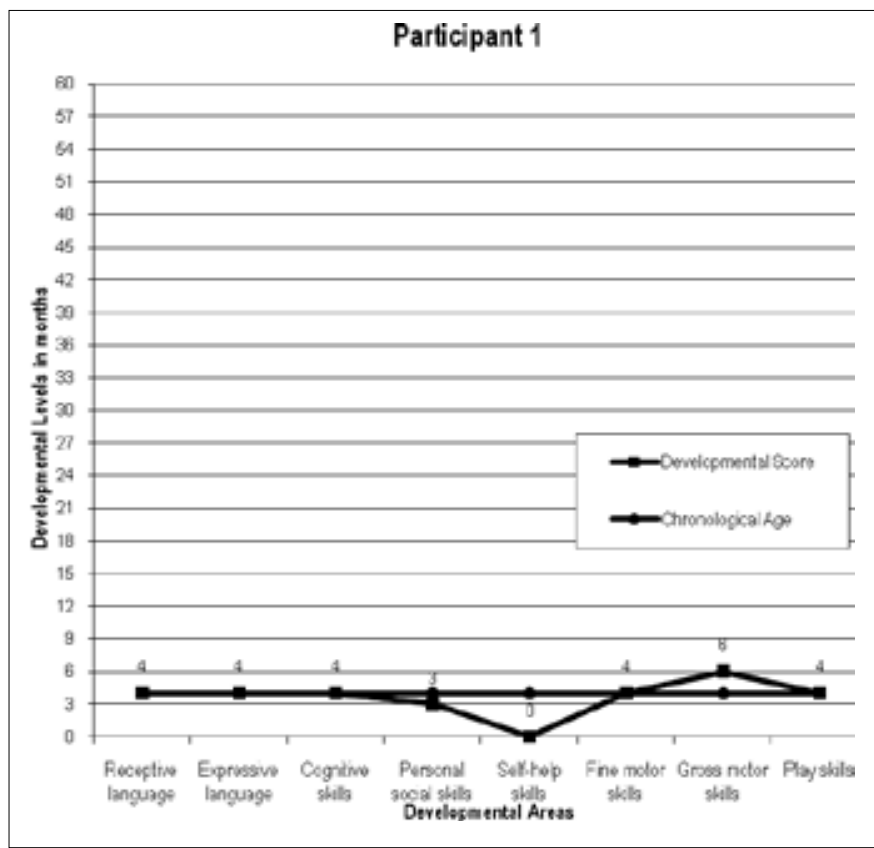

Fig. 1. Participant 1: Comparison between chronological age and developmental level of functioning.

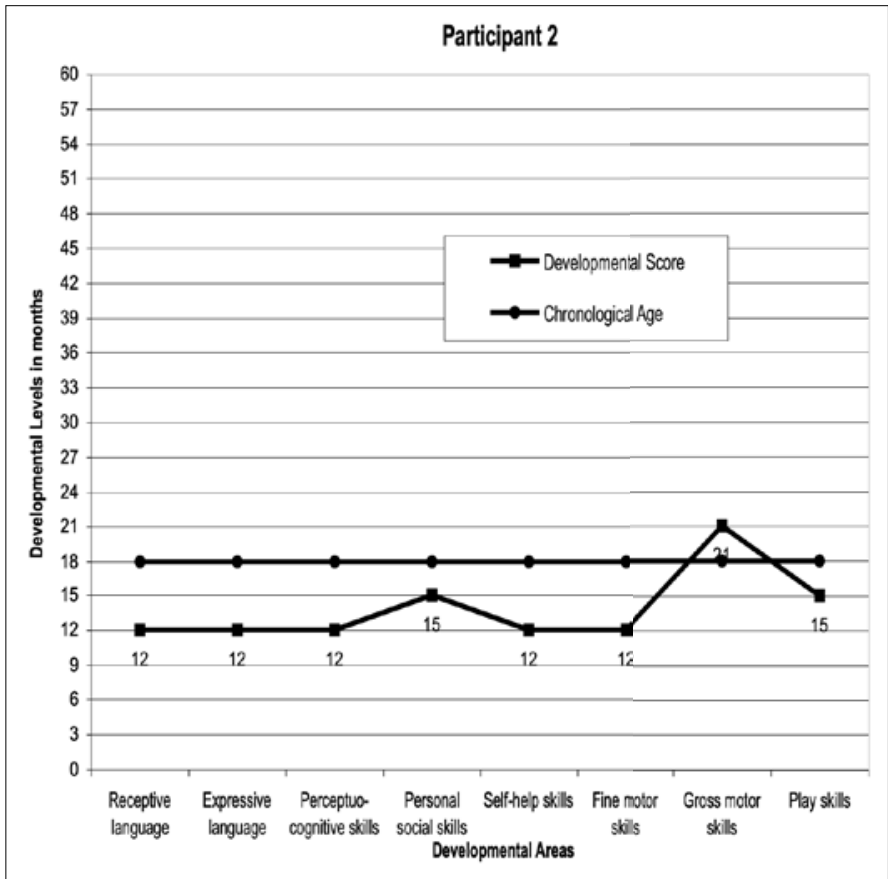

Fig. 2. Participant 2: Comparison between chronological age and developmental level of functioning.

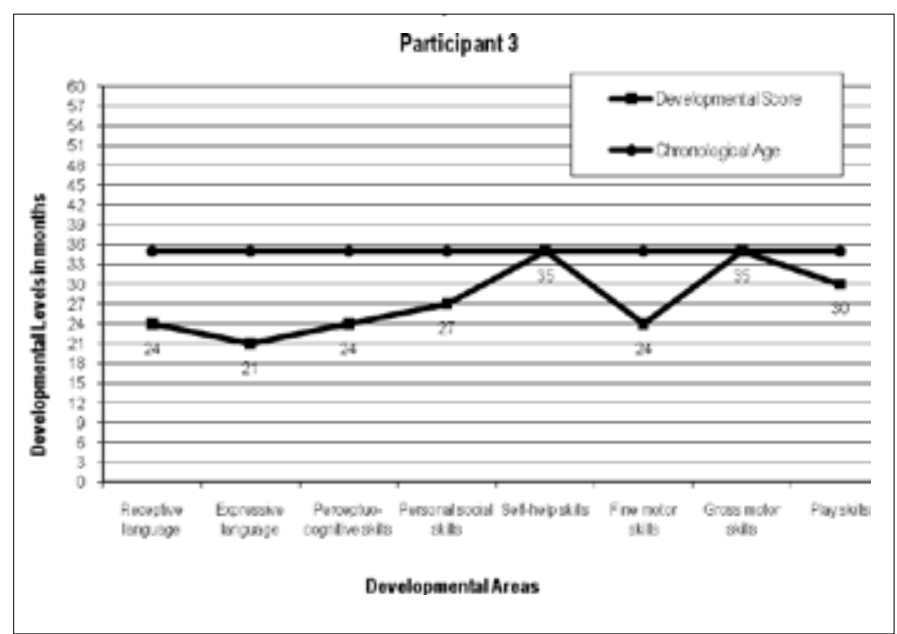

Fig. 3. Participant 3: Comparison between chronological age and participant's level of developmental functioning.

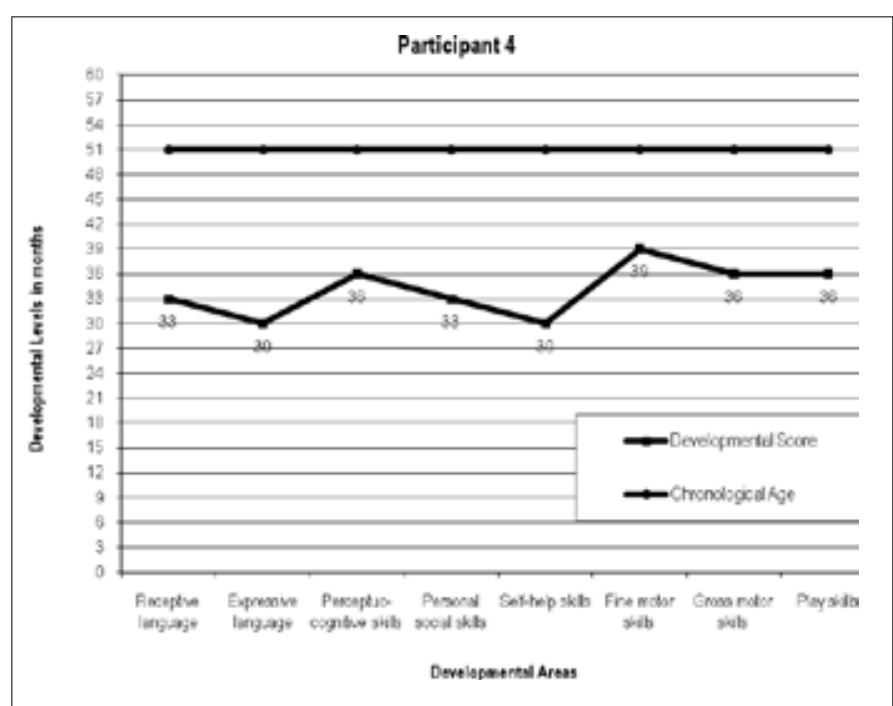

Fig. 4. Participant 4: Comparison between chronological age and participant's level of developmental functioning.

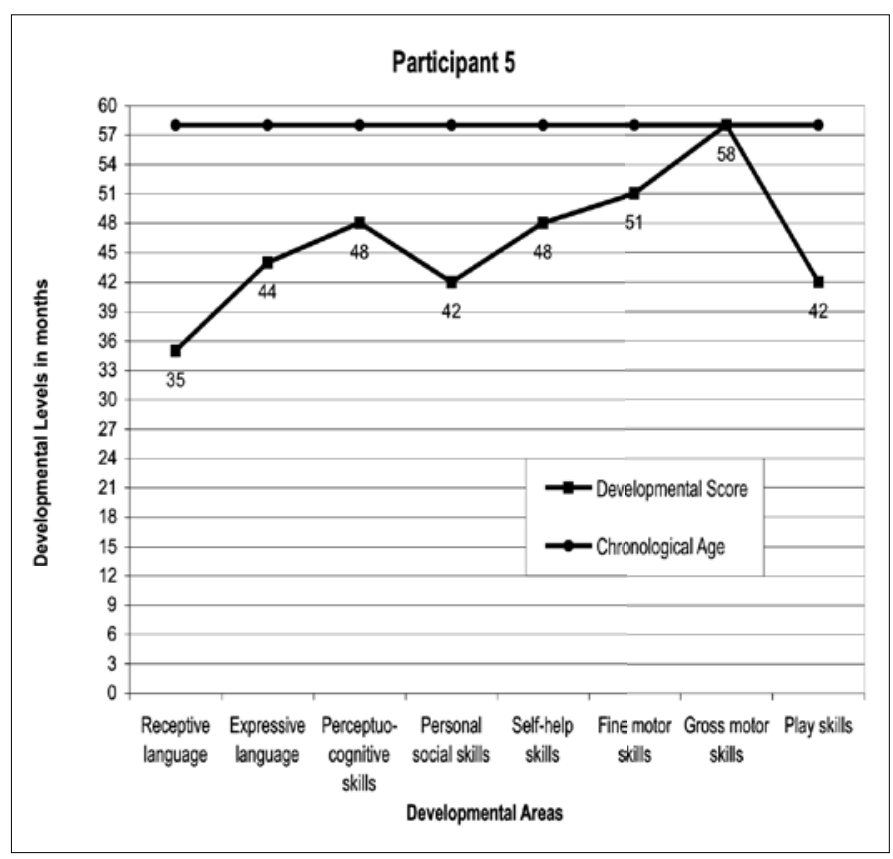

Fig. 5. Participant 5: Comparison between chronological age and participant's level of developmental functioning. 
The delay is considered moderate when a child is performing more than 6 - 12 months below chronological age expectations, and severe when a child is performing more than 15 months below chronological age expectations in developmental skill mastery. The individual profiles of each participant obtained in the research study are presented, from the youngest to the oldest participant, in Figures 1 to 5.

Language impairment, communication delay, verbosity and possible processing disorders were present in all the participants. The results correspond with those of Kodituwakku, Coriale, Fiorentino, Aragon, Kalberg, Buckley, Gossage, Ceccanti and May (2006), who identified language impairments as a primary distinguishing characteristic in a group of children with FASD in an epidemiological study in South Africa. Research by Coggins, Timler and Olswang (2007) indicated a key deficit among children with FASD - difficulty in using language in sophisticated social contexts. The results of the study also confirmed severe communication impairments as the area of key deficit. The participants also presented with impairment in responding and interacting appropriately in different social contexts such as parentchild interaction, emergent literacy activities and free play.

Furthermore, to confirm the evidence of language impairments in infants and young children with FASD, voxel-based morphometry data showed anomalies in the temporoparietal cortices in the left hemisphere, which are the critical areas for language processing (Kodituwakku et al., 2006). Speech-language therapy is therefore most important for children with FASD, as language functioning is a predictor of school success (Rossetti, 2001). Individual therapy may provide the SLT with a unique opportunity to provide safety in a therapeutic relationship and to model effective communication skills to enhance skill building in communication and related aspects in a young child with FASD (Henry, Sloane \& BlackPond, 2007). According to Henry et al. (2007), language impairments in particular, are likely to contribute to the children's poor coping strategies that often lead to negative and undesirable behaviour. Language impairments will therefore impact on the areas of academic, behavioural and social interactive competence in young children with FASD.

The results indicated that the group of participants was not homogeneous, although similarities were found in their profiles. The developmental profile of the youngest participant is indicative of a still emerging spectrum disorder; the full expression of delays may not yet be apparent at 4 months of age.

Participant 4 presented with multiple impairments and also showed the greatest delay in development. The three similar impairments observed in the participants were difficulties regarding social cognition, language and executive functioning. This triad of impairments is also described by Coggins et al. (2007).

The developmental profiles of the individual participants were combined to present a collective case study of the results of the five participants. The developmental trend that emerged from the results of the collective case study is depicted in Figure 6.

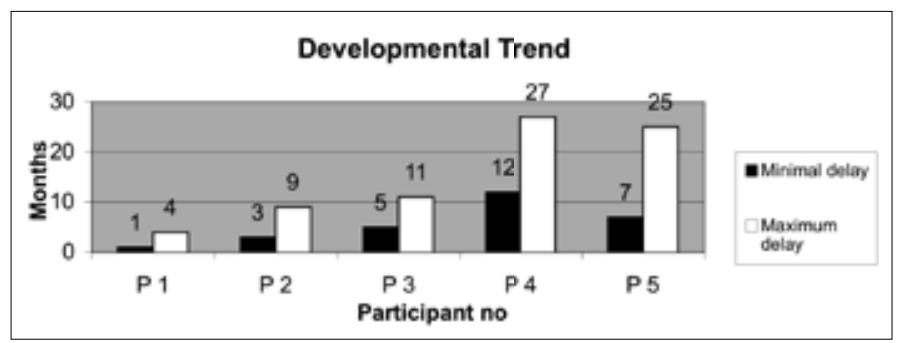

Fig. 6. Collective developmental trend emerging from the five participants.

It was evident from Figure 6 that the delays and impairments present in the participants became more pronounced with an increase in age. This trend corresponds with the findings of Henry et al. (2007), indicating that secondary disabilities associated with FASD often become more impaired as the individual ages. There was an increase in the greatest delays and a decrease in the minimal delays, indicating that the severity of delays was increasing. The trend was only visible with the combined profiles of the five participants and does not necessarily indicate greater delays with age for all infants and young children with FASD. None of the participants received any form of ECI and therefore the trend could differ for infants and young children with FASD who receive timely ECI. A definitive trend can, however, only be determined by means of a longitudinal study (Leedy \& Ormrod, 2005). To minimise the severe impact of FASD on infants and young children with FASD and their families, timely ECI is strongly indicated (Rogers-Adkinson \& Stuart, 2007). Timely ECI is important because developmental plasticity is still present to facilitate developmental gains and to minimise the effect of the communication and general developmental impairments (Batshaw \& Conlon, 1997).

It is also clear from the results of the collective case study that prenatal alcohol exposure has an impact on core developmental processes such as the neurophysiological growth of the brain and nervous system, psychological development regarding personality formation, and social cognition and communication (Henry et al., 2007). Because of the effect of FASD on core developmental processes, a wide array of developmental domains are affected in infants and young children with FASD. The detailed profile of the communication development of a small group of infants and young children with FASD affirmed the wide array of developmental domains affected. It is also evident that the participants in the study presented with complex multiple neurodevelopmental needs and key deficits in the domain of communication and related skills, with far-reaching impact on their daily functioning, everyday family life and future academic success. Despite their complex needs, strengths that emerged among participants were their socially engaging nature and interest in other people. Furthermore, foster care placement and adoption will aid them in attaining more developmental gains as a safer and more nurturing environment facilitates integration into preschool and community settings.

\section{Conclusion}

The participants in the study are part of an international increasing clinical population of infants and young children who require ECI (Coggins et al., 2007; Rossetti, 2001). Despite the increase, improved services to address the impairments and the social impact of FASD in the South African context are not readily available (Viljoen, 1999). Therefore multidisciplinary teams have a role to play in prevention of FASD in high-risk populations such as adolescents where alcohol abuse and teenage pregnancy are rife, as well as assessment and treatment of FASD. All SLTs and audiologists should revisit their caseloads to screen for children with mental disability without definitive aetiologies, to check for the possible presence of FASD.

Children with FASD are a unique group of clients and present with complex histories and a diversity in cultural backgrounds and clinical profiles (Rogers-Adkinson \& Stuart, 2007). SLTs working in the field of ECI in South Africa should equip themselves with specialist skills and knowledge to provide contextually relevant services to young children with FASD and their families. Undergraduate training at tertiary institutions should aid SLTs and audiologists to equip them with increased theoretical knowledge about FASD and clinical exposure to cases.

As a result of complex needs, families affected by FASD require interdisciplinary support by team members such as the social worker, educational psychologist, SLT, occupational therapist, audiologist, dietician, nurse and a variety of medical specialists.

Because of the permanent nature of FASD, continued services will be required after the ECI period through preschool, primary school, adolescence and adulthood (Streissguth, Barr, Kagan \& Bookstein, 1997). The characteristics of the school-aged population of children with FASD are more widely published (Sparks, 2000; Thorne, Coggins, Carmichael Olson \& Astley, 2007) than research regarding the early years of infancy and toddlerhood. Further extensive ECI research is necessary to enhance appropriate ECI service delivery to this population, and to 
contribute to the description of the complex communication profile of infants and young children with FASD.

As far as we know, this study was one of the first exploratory studies to describe the developmental histories, family/caregiving contexts and communication functioning of young children with FASD in South Africa. The design of the structured interview schedule and the adaptation of the early communication assessment protocol (Kritzinger \& Steenkamp, 2006) provided useful instruments for obtaining information regarding the infant or young child with FASD's development and family/caregiving context. These instruments were also useful to describe the communication functioning of the participants within a four-level EC framework that included the child's: (i) physical characteristics, pre- and perinatal history, sensory integrity; (ii) communication and related skills; (iii) general development; and (iv) risk factors and resilience.

Although the instruments comprising the four-level EC framework were useful, it can be challenging to assess children of diverse language and cultural backgrounds because of the lack of availability of trained translator services in the health care sector in South Africa, and the lack of adequate knowledge regarding communication practices of some indigenous cultures. The study posed some limitations regarding the use of a small sample size restricted to a specific geographical location and only including caregiver and child participants who were proficient in English and Afrikaans. To increase the generalisability of results of this type of study, a repeat of this exact study on a larger and more linguistic and culturally representative sample is therefore recommended.

\section{Acknowledgements}

The article is based on the postgraduate research project by Mari de Beer, 2009: 'Communication profiles of a group of young children $(0$ - 5 years) with fetal alcohol spectrum disorder' (unpublished Master's dissertation, Department of Communication Pathology, University of Pretoria). The study was financed in part by a postgraduate bursary from the University of Pretoria.

\section{References}

Abkarian, G. G. (1992). Communication effects of prenatal alcohol exposure. Journal of Communication Disorders, 25, 221-240.

Antoniadis, A., \& Daulton, D. (1992). Meeting the needs of children in Pennsylvania who are exposed to alcohol and other drugs. Infant-Toddler Intervention. The Transdisciplinary Journal, 2(1), 53-62.

Arizona Revised Statutes (ARS) 15-761, 1-38. (2010). Tucson, AZ: Arizona State Department Legislature.

Batshaw, M. L., \& Conlon, C. J. (1997). Substance abuse: A preventable threat to development. In M. L. Batshaw (Ed.), Children with disabilities (4th ed.) (pp. 143-162). Baltimore, MD: Paul H. Brookes Publishing.

Carmichael Olson, H., \& Burgess, D. M. (1997). Early intervention for children prenatally exposed to alcohol and other drugs. In M. J. Guralnick (Ed.), The effectiveness of early intervention (pp. 109-145). Baltimore, MD: Paul H. Brookes Publishing.

Carmichael Olson, H., Jirikowic, T., Kartin, D., \& Astley, S. (2007). Responding to the challenge of early intervention for fetal alcohol spectrum disorders. Infants \& Young Children, 20(2), 172-189.

Clarren, S., \& Astley, S. (1997). Development of the FAS diagnostic and prevention network in Washington State. In A. Streissguth \& J. Kanter (Eds.), The challenge of fetal alcohol syndrome: Overcoming secondary disabilities (pp. 40-51). Seattle: University of Washington Press.

Clarren, S. K., Carmichael Olson, H., Clarren, S. G. B., \& Astley, S. J. (2000). A child with fetal alcohol syndrome. In M. J. Guralnick (Ed.), Interdisciplinary clinical assessment of young children with developmental disabilities (pp. 307-326). Baltimore, MD: Paul H. Brookes Publishing.

Coggins, T. E., Timler, G. R., \& Olswang, L. B. (2007). A state of double jeopardy: Impact of prenatal alcohol exposure and adverse environments on the social communicative abilities of school-age children with fetal alcohol spectrum disorder. Language, Speech and Hearing Services in Schools, 38(2), 117-127.

Colangelo, W., \& Jones, D. G. (1982). The fetal alcohol syndrome: A review and assessment of the syndrome and its neurological sequelae. Progress in Neurobiology, 19(2), 271-314.
De Beer, M. (2009). The communication profiles of young children (0 - 5 years) with foetal alcohol spectrum disorder. Unpublished Master's thesis, University of Pretoria.

Department of Health. (2001). Fetal alcohol syndrome among world's highest in SA. First international conference in the developing world on birth defects and disabilities. Sandton, Johannesburg, South Africa. Retrieved from http://www.doh.gov.za/docs/ $\mathrm{pr} / 2001 / \mathrm{pr} 0906 \mathrm{c} \cdot \mathrm{html}$

De Vos, A. S. (2002). Qualitative data analysis and interpretation. In A. S. de Vos (Ed.), Research at grass roots: For the social sciences and human service professions (2nd ed.) (pp. 339-255). Pretoria: Van Schaik.

Dittmer, C. D., \& Lentz, S. (2004). Fetal alcohol syndrome. Retrieved on 28 January 2005 from http://www.emedicine.com/PED/topic767.html

Garbarino, J., \& Ganzel, B. (2000). The human ecology of early risk. In J. P. Shonkoff \& S. J. Meisels (Eds.), Handbook of early childhood intervention (2nd ed.) ( pp. 76-93). Cambridge: Cambridge University Press.

Guralnick, M. J. (1997). Second-generation research in the field of early intervention. In M. J. Guralnick (Ed.), The effectiveness of early intervention (pp. 3-20). Baltimore, MD: Paul H. Brookes Publishing.

Henry, J., Sloane, M., \& Black-Pond, C. (2007). Neurobiology and neurodevelopmental impact of childhood traumatic stress and prenatal alcohol exposure. Language, Speech and Hearing Services in Schools, 38(2), 99-108.

Kodituwakku, P., Coriale, G., Fiorentino, D., Aragon, A. S., Kalberg, W. O., Buckley, D., Gossage, J. P., Ceccanti, M., \& May, P. A. (2006). Neurobehavioural characteristics of children with fetal alcohol spectrum disorders in communities from Italy: Preliminary results. Alcoholism: Clinical and Experimental Research, 30(9), 1551-1561.

Kritzinger, A., \& Louw, B. (2002). A comprehensive assessment protocol for infants and toddlers at risk for communication disorders, Part II: Assessment protocol. CLINICA: Applications in Clinical Practice of Communication Pathology, Monograph 7, 3-17.

Kritzinger, A., \& Steenkamp, L. (2006). Communication development of a young child with fetal retinoid syndrome: A seven year follow-up study. The South African Journal of Communication Disorders, 53, 39-48.

Leedy, P. D., \& Ormrod, J. E. (2005). Practical research: Planning and design (8th ed.). Englewood Cliffs, NJ: Prentice Hall.

Ntsabula, A. (2001). Human genetics policy guidelines for the management and prevention of genetic disorders, birth defects and disabilities. Pretoria, South Africa: Department of Health.

Rogers-Adkinson, D. L., \& Stuart, S. K. (2007). Collaborative services: Children experiencing neglect and the side effects of prenatal alcohol exposure. Language, Speech and Hearing Services in Schools, 38(2), 149-156.

Rossetti, L. M. (2001). Communication intervention. Birth to three (2nd ed.). San Diego, CA: Singular Thomson Learning.

Rossetti, L. M. (2006). The Rossetti infant-toddler language scale manual. East Moline, IL: LinguiSystems.

Sparks, S. N. (2000). Prenatal substance use and its impact on young children. In T. L Layton, E. R. Crais \& L. R. Watson (Eds.), Handbook of early language impairment in children: Nature (pp. 287-316). New York: Delmar Thomson Learning.

Sparks, S. N., \& Gushurst, C. (1997). Interactions of neonates and infants with prenatal cocaine exposure. In L. M. Rossetti \& J. E. Kile (Eds.), Early intervention for special populations of infants and toddlers (pp. 181-194). San Diego: Singular Publishing Group.

Steinhaussen, H., Willms, J., Winkler Metzke, C., \& Spohr, H. (2003). Behavioural phenotype in foetal alcohol syndrome \& foetal alcohol effects. Developmental Medicine \& Child Neurology, 45(3), 179-182.

Streissguth, A. P., Barr, H., Kagan, J., \& Bookstein, F. (1997). Primary and secondary disabilities in fetal alcohol syndrome. In A. Streissguth \& J. Kanter (Eds.), The challenge of fetal alcohol syndrome: Overcoming secondary disabilities (pp. 25-39). Seattle: University of Washington Press.

Thorne, J. C., Coggins, T. E., Carmichael Olson, H., \& Astley, S. J. (2007). Exploring the utility of narrative analysis in diagnostic decision making: Picture-bound reference, elaboration, and fetal alcohol spectrum disorders. Journal of Speech, Language and Hearing Disorders, 50, 459-474.

Vig, S., Chinitz, S., \& Shulman, L. (2005). Young children in foster care: Multiple vulnerabilities and complex service needs. Infants \& Young Children, 18(2), 147-160.

Viljoen, D. (1999). Fetal alcohol syndrome (Editorial). South African Medical Journal, 89, 959-960.

Viljoen, D., Craig, P., Hymbaugh, M. P. H., Boyle, C., \& Blount, S. (2001a). Fetal alcohol syndrome - South Africa, 2001. MMWR, 52, 660-662. Retreived from http://www.cdc. gov $/ \mathrm{mmwr} / \mathrm{preview} / \mathrm{mmwrhtml} / \mathrm{mm} 5228 \mathrm{a} 2 . \mathrm{htm}$

Viljoen, D., Croxford, J., Gossage, J. P., Kodituwakku, P. W., \& May, P. (2001b). Characteristics of mothers of children with FAS in the Western Cape Province of South Africa: A case control study. Journal of Studies on Alcohol, 62, 962-965.

Werner, E. E. (2000). Protective factors and individual resilience. In J. P. Shonkoff \& S. J. Meisels (Eds.), Handbook of early childhood intervention (2nd ed.) (pp. 115-132). Cambridge: Cambridge University Press. 


\section{Appendix A. Physical, medical and general developmental characteristics of children with FASD}

\section{Physical characteristics}

Craniofacial characteristics

- Cleft lip and/or palate

- Poor development of dentition

- Microcephaly

- Indistinct philtrum (smooth and/

or long)

- Thin upper lip and small lips

- Thinned upper vermillion

- Small teeth with faulty enamel

- Prominent lateral palatine ridges in

the mouth

- Epicanthal folds

- Low nasal bridge

- Anteverted nostrils

- Minor ear anomalies, e.g. posterior

rotation of the ears, poorly formed

concha

- Short, upturned nose

- Micrognathia (small mandible)

- Pre- or postnatal growth deficiency

regarding head circumference

- Hypoplasia of the maxilla

- Ptosis (downward displacement of

the upper eyelid)

between the eyelids)

- Strabismus, myopia and clinical

micropthalmia

Other physical characteristics

- Brachyclinodactyly (shortened 5th

finger)

- Anomalous palmar creases

- Sacral dimple
- Flat midface (hypoplasia)

- Blepharophimosis (reduced space

Developmental characteristics

Cognitive impairments

Brain and systemic defects

- Musculoskeletal defects

- Genito-urinary defects

- Immune deficiency

- Increased risk for seizures

- Muscular hypotonia

- Disturbance in brain neurochem-

istry in the following systems:

dopaminergic, noradrenergic, serotonergic, cholinergic, glutamatergic, GABAergic and histaminergic systems

Organ defects

- Cardiac (i.e. atrial or ventrical septal defect, occasionally tetralogy of Fallot)

- Liver

- Kidney

- Morphological changes in brain structure (especially in the corpus callosum)

Increased risk of infections

- Upper respiratory tract infections

- Otitis media

Sources: Antoniadis \& Daulton (1992); Batshaw \& Conlon (1997); Beirne-Smith, Ittenbach \& Patton (2002); Carmichael Olson (1995); Carmichael Olson \& Burgess (1997); Carmichael Olson et al. (2007); Chapman (2000); Colangelo \& Jones (1982); Jacobson, Jacobson, Sokol, Martier \& Ager (1993); Kim, Sugai \& Kim (1999); Mattson \& Riley (1997); Morrison \& Villarreal (1993); O’Malley \& Nanson (2002); Schoenbrodt \& Smith (1995); Shprintzen (2001); Sparks (1993); Steinhausen, Willms, Winkler Metzke \& Spohr (2003) - all cited in De Beer M (2009).

$$
\text { ment }
$$

- Attention impairment

- Memory impairment

- Complex learning impairment

- Central processing disorders; slower,

less efficient information processing

- Working memory problems

- Problems regarding social cognition

- Deficits in story memory

- Executive-function deficit in deduc-

tive reasoning

- Inconsistent problem-solving

strategies

- Visual-spatial processing deficits

Motor impairments

- Visual-motor integration delay

- Sensory motor integration problems

- Psychomotor maturation delay

- Fine motor dysfunction

- Gross motor dysfunction

Sensory impairments risk of sensorineural and conductive

hearing loss, auditory filtering

- Vision disorders, e.g. strabismus

- Sensory-seeking behaviour

- Sensory processing deficits
- Auditory disorders, e.g. increased
Behavioural difficulties

- Increased non-alert state in infants

- Sleep disturbances -poor sleep/

wake cycle

- Social-emotional delay

- Early onset of ADHD of the inatten-

tion subtype

- Hyperactivity

- Autistic-like behaviours

- Antisocial

- Disruptive

- Hyperkinetic behaviour

- Slow-to-warm temperament

- Response inhibition deficit

- Poor self-regulatory abilities (primary regulatory disorder present from birth)

- Off-task behaviour

- Less goal-directed

- Irritable

- Impulsive

- Easily frustrated

- Poor self-confidence

- Insecure

- Anxious

- Aggressive

- Difficulty establishing and main-

taining relationships with peers

- Decreased capacity to initiate and organise play
- Neonatal irritability 


\section{Appendix B. Characteristics of communication impairments of young children with FASD}

Expressive language

Developmental stage:

Infancy 0 - 24 months

- High-pitched cry

- Decreased vocalisation

- Delayed onset of first

words

- Limited lexicons

- Delayed imitations of

interactional partner's

language production

- Frequent use of gestures

- Phonological skills may be

age-appropriate or limited

Developmental stage: Early

childhood (24 - 60 months)

- Struggles with sequencing

during storytelling

- Difficulty with more com-

plex narrative skills

- Articulation impaired sec-

ondary to neurologically

based incoordination

- Fluency problems

- Echolalia

- Short mean length of ut-

terance

- Word-finding problems

- Syntactical errors

- Difficulty with more com-

plex syntax

- Language expression more

affected than language

comprehension

- Increased verbosity

- Most frequently used word

class are nouns
Receptive language

- No descriptions found in

the literature

- Poor comprehension

linked to level of cognitive impairment

- Struggles to follow instruc-

tions and directions

- Semantic errors

\section{Pragmatics}

- Poor mother-child attachment leading to a negative environment for the development of communication skills

- Inappropriate use of gestures

- Limited social interaction

- Autistic-like behaviours

- Perseveration on a topic

- Inappropriate use of

language as means of initiating and continuing social discourse

- Various language function problems (e.g. lack of communication repair)

- Difficulty taking listener's perspective into account

- Autistic-like behaviours (e.g. passive in interaction, not displaying need to communicate

- Inappropriate answering of questions

- Poor eye contact

- Poor turn taking

- Increased talkativeness (verbosity)
Oral-motor development

- Feeding difficulties

- Difficulty with sucking

associated with low muscle tone

- Weak sucking reflex

- Long latency to suck:

lengthened time to initiate successful sucking

- Difficulty to regulate the

basic feeding cycle

- If cleft palate is present, associated feeding difficulties can be expected

- Delayed achievement of feeding milestones

- Poor appetite and lack of interest in food

- Deficiency in one or more of the eight major valves along the vocal tract

- Poor coordination of articulators, may be a remnant of apraxic behaviour

- Compensatory articulation patterns secondary to cleft palate or velopharyngeal insufficiency
Auditory abilities

- Abnormalities in orienta-

tion to auditory stimuli

- $93 \%$ of infants with FASD

present with recurrent

otitis media with effusion

during the first 6 years

of life; this is the highest

reported otitis media with

effusion rate of any group

of children with disabilities

in the literature

- Difficulty regarding speech-sound discrimination

- Involuntary response

to noises due to lack of

habituation

- Increased incidence of sensorineural and conductive

hearing loss

- Prone to otitis media

- Involuntary response to noises due to lack of habituation

- High incidence of sensorineural hearing loss as well as conductive hearing loss

- Speech discrimination deficits

Sources: Abkarian (1992); Batshaw \& Conlon (1997); Carmichael Olson (1995); Carmichael Olson \& Burgess (1997); Carmichael Olson et al. (2007); Clarren et al. (2000); Coggins, Olswang, Carmichael Olson \& Timler (2003); Crites, Fischer, McNeish-Stengel \& Siegel (1992); Gerber (1998); Kim et al. (1999); Lesar (1992); O’Malley \& Nanson (2002); Rivers \& Hedrick (1992); Rivers \& Hedrick (1998); Schoenbrodt \& Smith (1995); Sparks (2000) - all cited in De Beer M (2009). 